\title{
RESISTÊNCIA MECÂNICA DO LIGAMENTO PATELAR APÓS A RETIRADA DO TERÇO MÉDIO COM INCISÃO LONGITUDINAL OU DUAS MINI-INCISÕES TRANSVERSAS
}

\author{
PATELLAR LIGAMENT STRENGTH AFTER REMOVAL \\ OF THE MIDDLE-THIRD THROUGH EITHER \\ ONE LONGITUDINAL INCISION OR TWO MINI-TRANSVERSE INCISIONS
}

Leandro Calil De Lazari', Cleber Antonio Jansen Paccola ${ }^{2}$

\section{RESUMO}

Objetivo: Avaliar a resistência e a regeneração do ligamento patelar após a retirada de enxerto para reconstrução do ligamento cruzado anterior, utilizando a técnica tradicional de incisão na pele única longitudinal e uma técnica de duas mini-incisões transversas, em ovinos. Métodos: Foram utilizadas 10 ovelhas. No joelho direito, realizamos a retirada do enxerto do ligamento patelar através do método tradicional e, no joelho esquerdo, através do método de duas incisões. Os animais ficaram em observação por seis meses. O conjunto (ligamento patelar, tíbia e a patela) foi adaptado à máquina de testes mecânicos para testes de resistência do ligamento. Resultados: Não foi encontrada diferença entre as duas técnicas propostas com relação à regeneração e resistência do ligamento patelar. Observamos, porém, o encurtamento do ligamento patelar nos dois grupos, e que a sutura da fascia superficialis do ligamento patelar não influiu na regeneração do ligamento patelar, segundo a histologia. Conclusão: A técnica utilizando duas incisões na pele apresenta os mesmos padrões de regeneração e resistência do ligamento patelar remanescente, quando comparada à técnica tradicional de incisão única longitudinal.

Descritores - Ligamento cruzado anterior; Ligamento patelar; Ovinos

\begin{abstract}
Objective: To evaluate the mechanical strength and regeneration of the patellar ligament after harvesting the middle-third for reconstruction of the anterior cruciate ligament, using the traditional technique of a single longitudinal skin incision or two small transverse incisions, in sheep. Methods: Ten sheep were used in this study. In the right knee we removed the graft by the traditional method, and in the left knee, using the two transverse incision method. The animals were observed for six months. The specimens (patellar, tibia and patella) were adapted to the mechanical test machine. Results: No difference was found between the two proposed techniques in relation to regeneration and mechanical strength of the patellar ligament. Shortening of the patellar ligament was also observed in the two groups, and suture of the superficial fascia of the patellar ligament did not influence the regeneration of the patellar ligament, according to the histology. Conclusion: The technique using two mini-incisions in the skin does not present the same levels of regeneration or mechanical strength of the remaining patellar ligament as the traditional technique of a single, longitudinal incision.
\end{abstract}

Keywords - Anterior cruciate ligament; Patellar ligament; Sheep

\footnotetext{
1 - Médico Ortopedista, Doutorando da Pós-Graduação da Faculdade de Medicina de Ribeirão Preto - USP.

2 - Professor Titular do Departamento de Biomecânica, Medicina e Reabilitação do Aparelho Locomotor da Faculdade de Medicina de Ribeirão Preto - USP.
}

Trabalho realizado no Departamento de Biomecânica, Medicina e Reabilitação do Aparelho Locomotor da Faculdade de Medicina de Ribeirão Preto - USP.

Correspondência: Leandro Calil De Lazari, Rua Dr. Álvaro Costa Couto, 50, apto. 1.201 - 14015-140 - Ribeirão Preto, SP - E-mail: calil.delazari@hotmail.com

Declaramos inexistência de conflito de interesses neste artigo 


\section{INTRODUÇÃO}

As reconstruções do ligamento cruzado anterior (LCA), usando o terço médio do ligamento patelar como enxerto, têm tido resultados funcionais bons. Contudo, a persistência de dor no local da retirada do enxerto, distúrbio de sensibilidade anterior no joelho e dificuldade ao ajoelhar, são problemas encontrados em 40 a 60\% dos pacientes submetidos à reconstrução do LCA, quando se usa a técnica tradicional de retirada do enxerto patelar, através de uma incisão única longitudinal ${ }^{(1)}$.

As técnicas minimamente invasivas estão sendo desenvolvidas em diversas áreas da cirurgia, com o objetivo de diminuir a agressão às partes moles e possibilitar a reabilitação o mais precoce e indolor possível, além de melhorar o aspecto estético da cicatriz.

Verificamos na literatura duas técnicas menos invasivas de retirada do enxerto do ligamento patelar para reconstrução do LCA: uma descrita por Kartus et al ${ }^{(1)}$ através de duas mini-incisões longitudinais e outra, descrita por Carneiro et $a l^{(2)}$ e Tsuda et $a l^{(3)}$ através de duas mini-incisões transversas. Ambas com o objetivo de diminuir a agressão às partes moles, evitar a lesão do ramo infrapatelar do nervo safeno e a dor anterior do joelho.

Existe preocupação com relação ao efeito da sutura da fascia superficialis na regeneração do ligamento remanescente. Encurtamento do tendão é fato relatado por diversos autores.

Adam et al $^{(4)}$ removeram o enxerto do ligamento patelar e não aproximaram a falha deixada pela remoção, mas suturaram a fascia superficialis. Eles relataram que houve encurtamento do ligamento patelar em todos os casos.

Krosser et $a l^{(5)}$ analisaram dois grupos: um, no qual foi aproximada a falha deixada pela retirada do enxerto do ligamento patelar, e outro, em que a falha não foi aproximada. Eles concluíram que não houve diferença significativa entre os dois grupos. Referiram ainda encurtamento do ligamento patelar nos dois grupos.

Na literatura são poucos os relatos de lesão do ligamento patelar após a retirada de enxerto. Lee et $a l^{(6)}$ relataram alguns casos de complicações pós-operatórias no ligamento patelar, com ruptura do ligamento, após a retirada do enxerto. No entanto, existe a questão se a dificuldade de visualização inerente à técnica de duas mini-incisões na remoção do enxerto patelar, poderia causar remoção além do desejado, e enfraquecendo o ligamento remanescente. Também permanece a questão se a sutura da fascia superficialis, possível apenas na técnica clássica de incisão longitudinal única, tem influência na regeneração do ligamento ${ }^{(1,7,8)}$.
O nosso objetivo neste trabalho foi analisar a resistência mecânica do ligamento patelar remanescente, após a retirada de enxerto (1/3 central), visando a reconstrução do ligamento cruzado anterior, removidos segundo a técnica tradicional (incisão longitudinal única) e duas mini-incisões transversas.

\section{MÉTODOS}

Foram utilizadas 10 ovelhas adultas deslanadas, sem raça definida, com peso de 45 a $55 \mathrm{~kg}$.

No joelho direito foi retirado o enxerto osso-tendãoosso da forma tradicional, por uma incisão longitudinal, do pólo inferior da patela até a tuberosidade anterior da tíbia (TAT). A fascia superficialis foi suturada e os terços remanescentes do ligamento foram aproximados com pontos separados. No joelho esquerdo foi realizada incisão através de duas mini-incisões, seguindo a largura da patela e da TAT (Figuras 1 e 2).

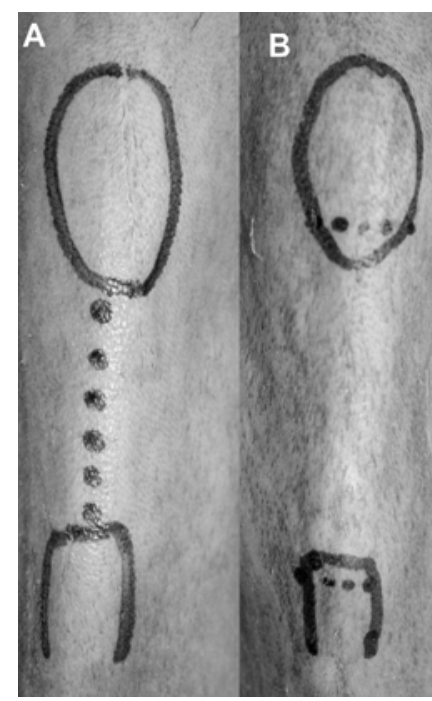

Figura 1 - Vista anterior do joelho. A linha pontilhada corresponde às incisões programadas. A) Incisão única longitudinal. B) Duas mini-incisões transversas

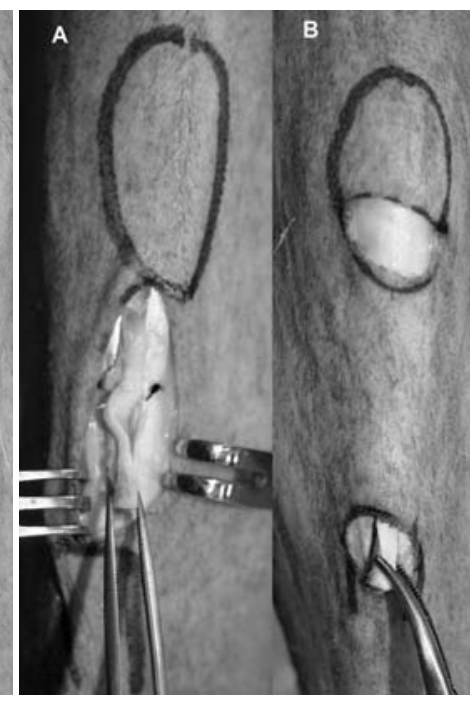

Figura 2 - Vista anterior do joeIho no intraoperatório. A) Incisão tradicional, enxerto já individualizado e bloco ósseo tibial já elevado. B) Duas incisões. A retirada se inicia de distal para proximal
Nas duas técnicas, as extremidades lateral e medial do ligamento patelar foram marcadas com pontos de fio de náilon 3-0, para posteriormente facilitar a localização destes pontos. Todos os ligamentos foram mensurados, anotando o comprimento, perímetros proximal, médio e distal, com aparelho especialmente desenvolvido para este fim. O modelo de mensuração está exemplificado na Figura 3 e os valores na Tabela 1. 

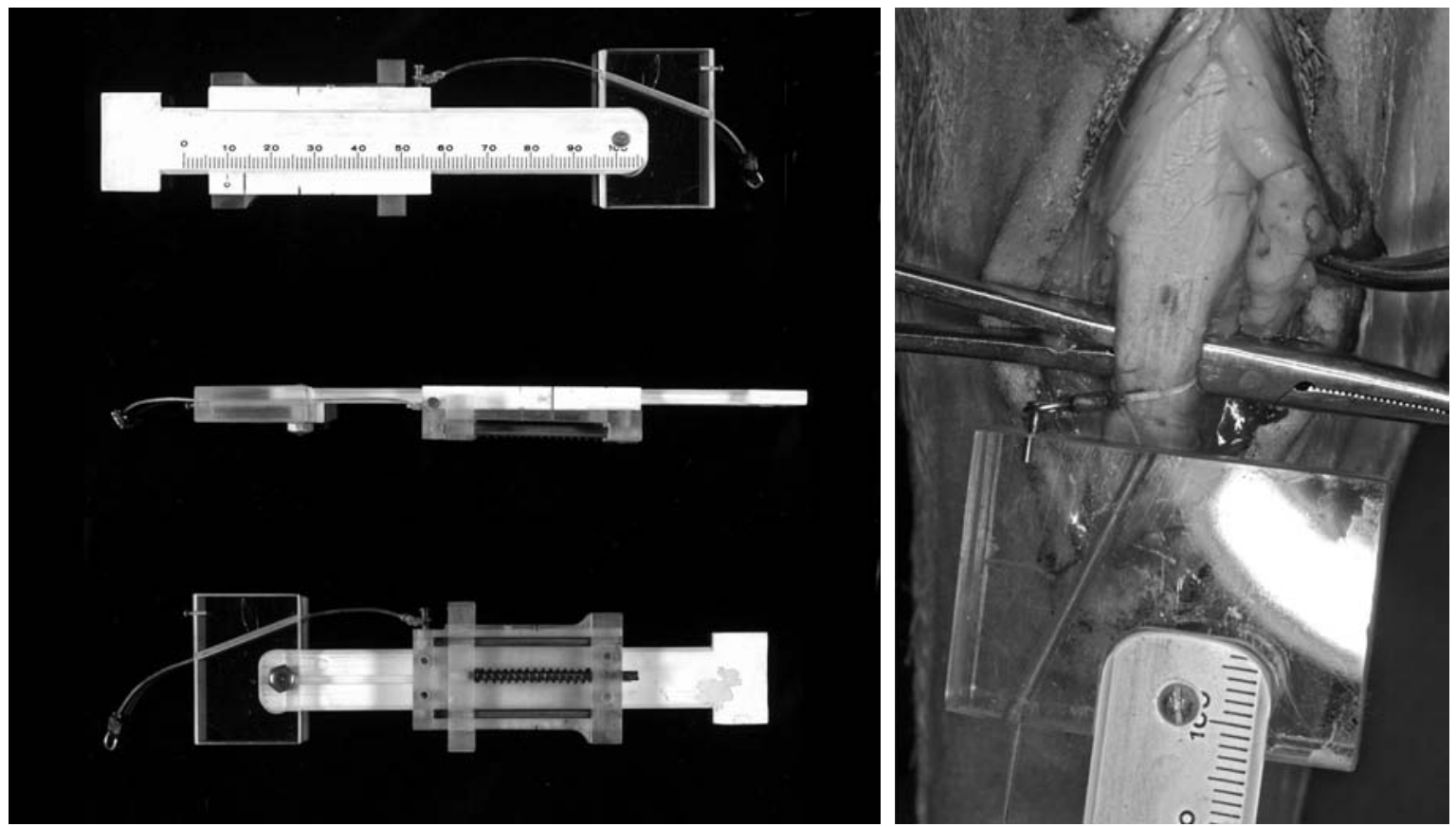

Figura 3 - Vista do instrumento para medida do perímetro e correspondente mensuração intraoperatória

Tabela 1 - Média dos perímetros, áreas e comprimentos dos ligamentos patelares

\begin{tabular}{c|c|c}
\hline $\begin{array}{c}\text { Técnica tradicional, } \\
\text { média dos valores }\end{array}$ & Cirurgia & Eutanásia \\
\hline Perímetro superior $(\mathrm{mm})$ & 20,18 & 30,36 \\
\hline Perímetro médio $(\mathrm{mm})$ & 19,55 & 29,45 \\
\hline Perímetro inferior $(\mathrm{mm})$ & 20,23 & 29,55 \\
\hline Comprimento longitudinal $(\mathrm{mm})$ & 54,64 & 35,27 \\
\hline Área superior $\left(\mathrm{mm}^{2}\right)$ & 32,60 & 74,40 \\
\hline Área média $\left(\mathrm{mm}^{2}\right)$ & 30,49 & 69,91 \\
\hline Área inferior $\left(\mathrm{mm}^{2}\right)$ & 32,74 & 70,25 \\
\hline & & \\
\hline Técnica duas incisões, & Cirurgia & Eutanásia \\
média dos valores & 19,77 & 29,09 \\
\hline Perímetro superior $(\mathrm{mm})$ & 19,55 & 29,45 \\
\hline Perímetro médio $(\mathrm{mm})$ & 19,55 & 28,00 \\
\hline Perímetro inferior $(\mathrm{mm})$ & 55,27 & 34,73 \\
\hline Comprimento longitudinal $(\mathrm{mm})$ & 31,18 & 68,36 \\
\hline Área superior $\left(\mathrm{mm}^{2}\right)$ & 30,49 & 69,91 \\
\hline Área média $\left(\mathrm{mm}^{2}\right)$ & 30,46 & 63,49 \\
\hline Área inferior $\left(\mathrm{mm}^{2}\right)$ & &
\end{tabular}

O perímetro da região média do ligamento patelar, na técnica das duas incisões (cirurgia), foi considerado igual ao do lado contralateral, pois se tratava do mesmo animal. Os animais ficaram em livre locomoção e suporte total de carga.

Após seis meses, os animais foram submetidos a eutanásia (período sugerido por Picado et $a l^{(7)}$ ), fotografadas as cicatrizes, os ligamentos patelares isolados e mensurados, seguindo as marcações dos pontos de náilon. Retirado o conjunto patela - ligamento patelar - tíbia dos animais, para os testes mecânicos e análise histológica.

Foram escolhidos sequencialmente, de forma alternada, cinco animais para análise histológica e cinco animais para os testes mecânicos.

O teste mecânico de tração foi realizado em máquina de testes*. Os testes utilizados foram: tração do ligamento patelar até a sua ruptura, força máxima para romper o ligamento e a tensão máxima do ligamento no momento da ruptura (Figura 4).

A análise histológica foi feita analisando a porção a meia distância patela-tíbia. Os cortes foram corados com hematoxilina-eosina e examinados com aumento de 25 vezes.

\section{RESULTADOS}

A análise estatística foi feita utilizando o teste não paramétrico de Wilcoxon, analisando a deformação, a força máxima e a tensão máxima no momento da ruptura do enxerto. Obtivemos um valor de p correspondentemente maior que $5 \%$ em todas as análises, o que indica não existir diferença significante entre os dois grupos (Tabela 2).

$\mathrm{Na}$ análise da área e do comprimento longitudinal do ligamento patelar na cirurgia e na eutanásia, o valor de p foi maior que 5\%, o que indica não haver diferença significativa entre os grupos. 


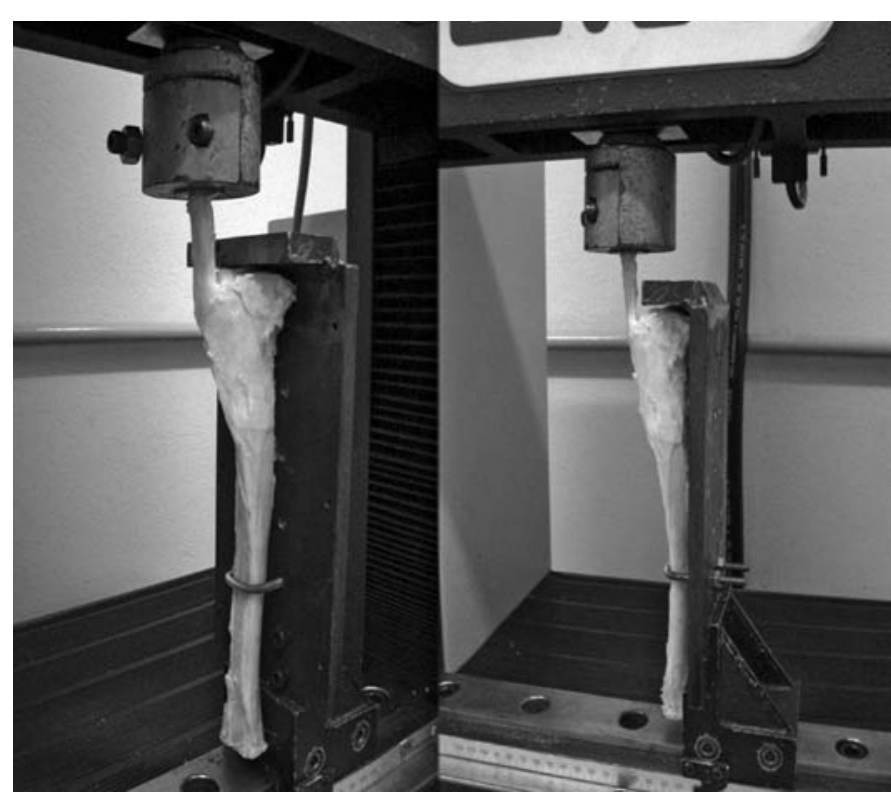

Figura 4 - Vista do conjunto patela - ligamento patelar - tíbia, na máquina de ensaio. *Modelo EMIC DL 10000 (São José dos Pinhais, Curitiba, PR, Brasil)

Tabela 2 - Análise estatística da deformação, força máxima e tensão máxima

\begin{tabular}{|c|c|c|c|c|c|}
\hline & Média & $\begin{array}{l}\text { Desvio } \\
\text { padrão }\end{array}$ & $\begin{array}{l}\text { Coeficiente } \\
\text { de variação }\end{array}$ & Mediana & $\begin{array}{l}\text { Valor } \\
\text { de } p\end{array}$ \\
\hline Deformação & $(\%)$ & $(\%)$ & $(\%)$ & $(\%)$ & \\
\hline Tradicional & 51,19 & 19,48 & 38,05 & 42,95 & \multirow{2}{*}{0,06} \\
\hline Duas incisões & 60,23 & 15,78 & 26,20 & 57,06 & \\
\hline Força máxima & (Newton) & (Newton) & (Newton) & (Newton) & \\
\hline Tradicional & 2221 & 843 & 37,99 & 2058 & \multirow{2}{*}{0,59} \\
\hline Duas incisões & 2415 & 450 & 18,64 & 2232 & \\
\hline Tensão máxima & (Pascal) & (Pascal) & (Pascal) & (Pascal) & \\
\hline Tradicional & 34532118 & 15256297 & 44,18 & 30744388 & \multirow{2}{*}{0,59} \\
\hline Duas incisões & 42082416 & 12939589 & 30,75 & 41500008 & \\
\hline
\end{tabular}

Nas medidas de comprimento, observamos o encurtamento do ligamento patelar e abaixamento da patela em todos os casos (Figura 5 e Tabela 1).

Comparando os comprimentos dos ligamentos patelares no intraoperatório e na eutanásia, concluímos que houve significativo encurtamento e aumento do perímetro do ligamento patelar do mesmo ligamento, mas não houve diferença entres as duas técnicas (Figura 5).

$\mathrm{Na}$ avaliação histológica, observamos perda da orientação paralelas das fibras, interposição de colágeno, pequena quantidade de vasos sanguíneos, e aspecto condroide do ligamento, mas não houve diferença perceptível entre as duas técnicas.

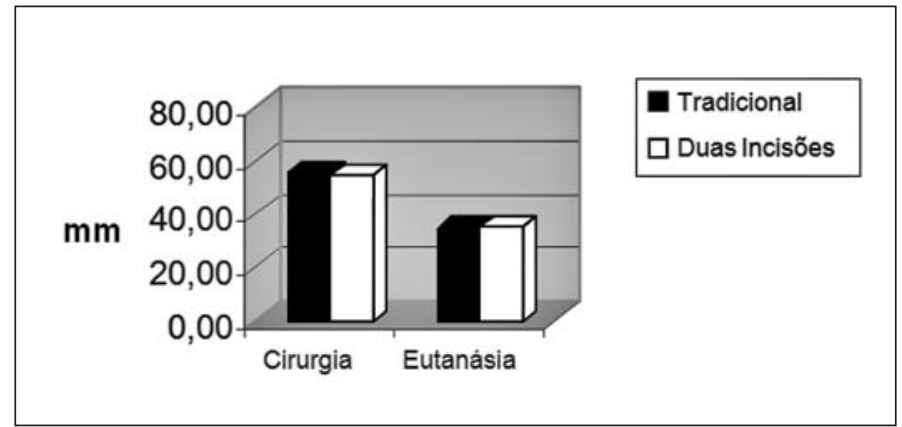

Figura 5 - Média do comprimento longitudinal do ligamento patelar no intraoperatório e na eutanásia. Houve importante encurtamento do ligamento, que ocorreu em ambas as técnicas, de forma semelhante

\section{DISCUSSÃO}

O desconforto da lesão do ramo infrapatelar do nervo safeno, que ocorre em muitos pacientes submetidos à retirada de enxerto do ligamento patelar através da técnica tradicional, justifica a tentativa de encontrar uma alternativa técnica. A possibilidade de melhoria na estética cicatricial e menor lesão vascular são outros pontos que nos motivaram a desenvolver e estudar uma nova técnica menos invasiva.

Algumas técnicas menos invasivas de retirada do enxerto do ligamento patelar foram publicadas na literatura $^{(1,2,3,9)}$, mostrando bons resultados nos seus objetivos.

A técnica de uma única incisão horizontal descrita por Portland et al $^{(10)}$ tem como objetivo evitar a lesão do ramo infrapatelar do nervo safeno. Porém, trata-se de uma única incisão longa e relativamente agressiva, com necessidade de descolamento extenso do subcutâneo e estiramento da pele para conseguir a retirada do enxerto.

Kartus et $a l^{(1)}$, Carneiro et $a^{(2)}$, Tsuda et $a l^{(3)}$ e Garofalo et $a^{(11)}$, demonstraram como as técnicas menos invasivas de retirada de enxerto usando duas mini-incisões podem resultar em benefício aos pacientes mostrando resultados animadores.

No entanto, estas técnicas levantam dúvida sobre a possibilidade de enfraquecer o ligamento remanescente. Pela dificuldade de visualização, o cirurgião poderia remover o enxerto excessivamente robusto, ou, ao contrário, muito delgado, afetando assim as propriedades mecânicas do ligamento remanescente.

A ruptura do ligamento patelar, após a retirada do enxerto, é uma complicação rara, mas relatada na literatura $^{(12)}$. O fato de estarmos propondo uma nova técnica, na qual não existe um controle preciso da retirada, além da não realização da sutura do peritendão, nos preocupou e nos motivou a realizar este estudo. 
Desta forma, o presente estudo é pertinente, pois ao pesquisar no PubMed, não foi encontrado artigo analisando repercussão na resistência do ligamento patelar remanescente, usando uma técnica menos invasiva. Mesmo assim, como é uma técnica pouco utilizada, a falta de relato de ocorrência de complicação não é prova de que não possa ocorrer o enfraquecimento excessivo associado à esta técnica de obtenção do enxerto.

Em nossos casos clínicos, observamos que a retirada do enxerto do ligamento patelar através das duas mini-incisões é mais fácil de ser realizada de distal para proximal, devido ao fato de que, na inserção do ligamento patelar na tíbia, ele é mais facilmente delimitável e mais fácil de ser visualizado. O isolamento do terço central do ligamento pode ser feito com um instrumental rombo tipo hemostático Kelly ou faca de Smillie para menisco. No início pode até ser um pouco difícil de realizar a técnica, mas, após alguns casos, ela se torna fácil e segura.

O não fechamento da fascia superficialis, na técnica minimamente invasiva, foi outro fator que nos preocupava relativamente à regeneração do ligamento patelar. Observamos, porém, que não houve diferença na regeneração tecidual, tanto do ponto de vista mecânico como histológico, mostrando assim que, aparentemente, a sutura da fascia superficialis não influencia no processo de reparação da falha criada no ligamento.

Foi observado aumento do perímetro do ligamento patelar, observado em ambos os grupos (Tabela 1). Isto pode ser devido ao encurtamento do ligamento patelar, mas sem dúvida, não associado à técnica empregada de remoção do enxerto.

\section{REFERÊNCIAS}

1. Kartus J, Ejerhed L, Sernert N, Brandsson S, Karlsson J. Comparison of traditional and subcutaneous patellar tendon harvest. A prospective study of donor site-related problems after anterior cruciate ligament reconstruction using different graft harvesting techniques. Am J Sports Med. 2000;28(3):328-35

2. Carneiro Filho $M$, Navarro RD, Cortelazo MJ. Reconstrução artroscópica do ligamento cruzado anterior com tendão patelar utilizando dupla incisão anterior transversa. Rev Bras Ortop. 1997;32(4):279-82.

3. Tsuda E, Okamura Y, Ishibashi Y, Otsuka H, Toh S. Techniques for reducing anterior knee symptoms after anterior cruciate ligament reconstruction using a bone-patellar tendon-bone autograft. Am J Sports Med. 2001;29(4):450-6.

4. Adam F, Pape D, Kohn D, Seil R. Length of the patellar tendon after anterior cruciate ligament reconstruction with patellar tendon autograft: a prospective clinical study using Roentgen stereometric analysis. Arthroscopy. 2002;18(8):859-64.

5. Krosser $\mathrm{BI}$, Bonamo JJ, Sherman $\mathrm{OH}$. Patellar tendon length after anterior cruciate ligament reconstruction. A prospective study. Am J Knee Surg. 1996;9(4):158-60

6. Lee GH, McCulloch P, Cole BJ, Bush-Joseph CA, Bach BR Jr. The incidence of acute patellar tendon harvest complications for anterior cruciate ligament reconstruction. Arthroscopy. 2008;24(2):162-6.

7. Picado CHF, Paccola CAJ. Efeitos do enfraquecimento e da desvitalização do ligamento patelar de ovinos sobre sua resistência à tração. Rev Bras Ortop. 1996;31(9):759-70
O fator estético também é uma preocupação na reconstrução do $\mathrm{LCA}^{(13)}$. Ao observar as cicatrizes das duas técnicas cirúrgicas realizadas, ficou evidente que a técnica das duas incisões é muito mais estética que a técnica tradicional. Isto seria provavelmente porque na técnica das duas incisões, as incisões são paralelas às linhas de rugas da pele, sendo por isto mais estéticas e menos evidentes.

Muellner et al $^{(14)}$ referiram o abaixamento da patela em $30 \%$ dos casos. No nosso estudo, o encurtamento do ligamento patelar foi bastante grande (Tabela 1) e ocorreu em todos os casos. A razão do encurtamento é discutível, mas, sem dúvida, não foi devido ao repouso pós-operatório, uma vez que os animais estavam soltos e caminhando logo após a cirurgia.

Do ponto de vista mecânico, observamos que a ruptura nos testes do ligamento, aconteceu no terço proximal na maioria dos casos e junto à patela. Isto aconteceu de forma semelhante nos dois grupos. Busfield et $a l^{(15)}$, em um trabalho com pacientes submetidos a revisão do LCA, relataram um caso de ruptura junto à patela, quando fazia a retirada do enxerto do ligamento patelar contralateral. Isto pode sugerir que o ponto mais fraco, após a retirada do enxerto do ligamento patelar, é a inserção proximal na patela, o que está de acordo com nossas observações.

\section{CONCLUSÃO}

Não houve diferença significante na resistência do ligamento patelar entre as duas técnicas analisadas.

Não observamos diferença histológica na regeneração do ligamento patelar entre as duas técnicas analisadas.

8. Rogers GJ, Milthorpe BK, Muratore A, Schindhelm K. Measurement of the mechanical properties of the ovine anterior cruciate ligament bone-ligament-bone complex: a basis for prosthetic evaluation. Biomaterials. 1990;11(2):89-96.

9. Drain O, Beaufils P, Thevenin LC, Boggione C, Katabi M, Charrois O et al. [Miniinvasive double-incision for patellar tendon harvesting in anterior cruciate ligament reconstruction]. Rev Chir Orthop Reparatrice Appar Mot. 2007;93(8):836-41.

10. Portland GH, Martin D, Keene G, Menz T. Injury to the infrapatellar branch of the saphenous nerve in anterior cruciate ligament reconstruction: comparison of horizontal versus vertical harvest site incisions. Arthroscopy. 2005;21(3):281-5.

11. Garofalo R, Mouhsine E, Chambat P, Siegrist O. Anatomic anterior cruciate ligament reconstruction: the two-incision technique. Knee Surg Sports Traumatol Arthrosc. 2006;14(6):510-6

12. Marumoto JM, Mitsunaga MM, Richardson AB, Medoff RJ, Mayfield GW. Late patellar tendon ruptures after removal of the central third for anterior cruciate ligament reconstruction. A report of two cases. Am J Sports Med. 1996;24(5):698-701.

13. Levy $\mathrm{HJ}$, Byck DC. Patellar tendon autograft harvesting using a mini vertical incision. Arthroscopy. 2000;16(5):558-62.

14. Muellner T, Kaltenbrunner W, Nikolic A, Mittlboeck M, Schabus R, Vecsei V. Shortening of the patellar tendon after anterior cruciate ligament reconstruction. Arthroscopy. 1998;14(6):592-6.

15. Busfield BT, Safran MR, Cannon WD. Extensor mechanism disruption after contralateral middle third patellar tendon harvest for anterior cruciate ligament revision reconstruction. Arthroscopy. 2005;21(10):1268 